\title{
Cryogenic optics for space applications
}

Natacha Cocheteau, Julien Basile, Bruno Delaborde, Matthieu Gilles, Patrick Robert, et al.

Natacha Cocheteau, Julien Basile, Bruno Delaborde, Matthieu Gilles, Patrick Robert, Mailys Thorigny, "Cryogenic optics for space applications," Proc. SPIE 11852, International Conference on Space Optics - ICSO 2020, 118522F (11 June 2021); doi: 10.1117/12.2599378

SPIE Event: International Conference on Space Optics - ICSO 2021, 2021, Online Only 


\section{International Conference on Space Optics-ICSO 2020}

Virtual Conference

30 March-2 April 2021

Edited by Bruno Cugny, Zoran Sodnik, and Nikos Karafolas
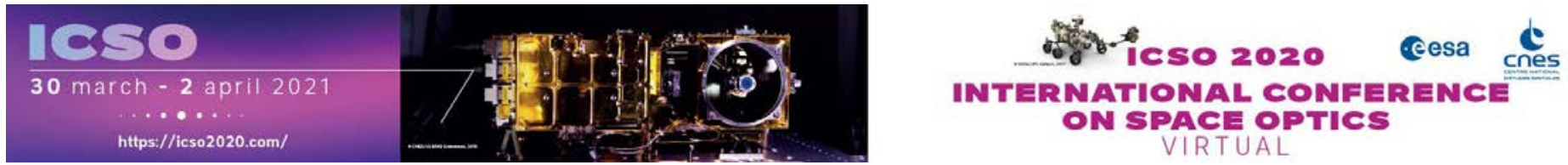

\section{Cryogenic optics for space applications}

\section{Cesa isopueations ecnes}

International Conference on Space Optics - ICSO 2020, edited by Bruno Cugny, Zoran Sodnik, Nikos Karafolas, Proc. of SPIE Vol. 11852, 118522F - (c) 2021 ESA and CNES

CCC code: $0277-786 \mathrm{X} / 21 / \$ 21 \cdot$ doi: $10.1117 / 12.2599378$ 


\title{
Cryogenic optics for space applications
}

\author{
Natacha Cocheteau $^{*}$, Julien Basile ${ }^{\mathrm{a}}$, Bruno Delaborde ${ }^{\mathrm{a}}$, Matthieu Gilles ${ }^{\mathrm{a}}$, Patrick Robert ${ }^{\mathrm{a}}$, Mailys \\ Thorigny $^{\mathrm{a}}$ \\ ${ }^{a}$ Thales-SESO, 530 rue Frédéric Joliot 13290 Aix-les-Milles, France
}

\begin{abstract}
For space Astronomy as well as Earth Observation from space, more and more focal plane instruments are operating in the near or mid infrared and require optics operating at cryogenic temperature. The challenge here is to design an optical system reaching all optics, mechanics and thermal requirements.

Thales SESO is presently under manufacturing of different opto-mechanical sub-assemblies for Meteosat Third Generation program. The imager missions MTG-I will produce images of the Earth simultaneously in different spectral channels, ranging from the visible spectrum to thermal infra-red, in order to fulfill the scientific, governmental \& population needs. The sounder mission MTG-S addresses some interferometric measurements in the MWIR and LWIR ranges on the same instrument.

The four CO-I objectives and the four one of CO-S work in different infrared ranges, thus, materials and coating have been chosen and designed especially for each spectral group. Each objectives is a stack of lenses bonded into barrels, themselves bonded inside a main barrel. Each objective is optimized based on the opto-mechanical values measured on each component. The objectives need to resist to mechanical and thermal environments in terms of opto-mechanical stability and work under vacuum, thus, with cleanliness specifications. All these requirements need to be taken into account on the design of different part of the cold optics.

Through this on-going realization, the main technical issues and corresponding solutions in terms of design, manufacturing and testing of lens assemblies, mirrors and relevant optical coatings will be pointed out.
\end{abstract}

Keywords: cryogenic optics, infrared objectives, coating, opto-mechanical design

\section{INTRODUCTION}

With the third generation of satellite (MTG), EUMETSAT and ESA innovate on observation satellite (FCI) with fixed satellites and new performances.

The previous meteorological satellite, MSG, is a satellite in constant rotation able to obtain:

- $\quad$ Full images with high resolution every 15 min

- $\quad$ Detailed images (Europe) every 5 minutes

- A resolution of $1 \mathrm{~km}$ and $3 \mathrm{~km}$ depending on the spectral range.

As for MTG, which is a fixed satellite with a more complex thermal environment, it will be able to obtain:

- $\quad$ Full images with high resolution every 10 minutes

- $\quad$ Detailed images (Europe) every 2.5 minutes

- A resolution of $0.5-1 \mathrm{~km}$ and $1-2 \mathrm{~km}$ depending on the spectral range

- 16 spectral ranges (grouped in 5 channels) instead of 12 (MSG)

The imager missions MTG-I will produce images of the Earth simultaneously in different spectral channels, ranging from the visible spectrum to thermal infra-red, in order to fulfill the scientific needs.

In the main MTG-I telescope output, there is a spectral separation into 5 channels:

- VIS: without changing the focal length of the telescope

- $\quad$ NIR, IR1, IR2, and IR3: by collimating the 4 corresponding output beams, and adapting the magnification ratio in order to achieve the required instrument focal length for each channel. This is made thanks to 4 different Cold Optics lens assemblies that have been developed by Thales SESO. 
In parallel Thales SESO also developed the objectives of the MTG Infrared Sounder (IRS) but this paper will only focus on design development for FCI Cold Optics.

The Cold Optics functional temperature is $80^{\circ} \mathrm{K}$ in the Infrared. Thales SESO realized the optical design, the optomechanical design as well as the anti-reflective coating design of the lenses.

Challenges with optomechanical system operating around ambient temperature/pressure compared to an optomechanical system operating under cryogenic environment and possibly in vacuum are very different:

- The optical (refractive index, dn/DT, CTE, homogeneity...) and mechanical (CTE, Young modulus...) material properties at both ambient and cryogenic temperatures need to be acknowledge

- Design solution have to be compatible with regard to mechanical stress occurring with temperature changes and gradient.

- Coatings need to keep good spectrophotometric performances for wide range of temperature and wavelength while offering specific adhesion, cleanability and durability properties on a large range of substrates with significant difference of relative CTE

- The demonstration of the final optical performances at operating conditions requiring complex test set-up in simulated environment, i.e. cryogenic test chamber with optical window and different on-board instrumentation with its own qualification in terms of optical performances.

Conception of a cryogenic objective is linked to four domains of knowledge which are thermic, mechanics, optics and optical coating. Each of them has an impact on the other one.

In this paper will be presented the major phases of the design conception, followed by the description of the integration and measurement phase. To finish will be presented the cryogenic tests and theirs results.

\section{DESIGN CONCEPTION}

The MTG Cold Optics objectives are operating at $80^{\circ} \mathrm{K}$ with their full performances. Thus, challenges in terms of design, simulations, manufacturing coating and testing are the following:

- Design: the objectives will be operating under vacuum at $80^{\circ} \mathrm{K}$, thus solutions in terms of material, stacking, bonding and venting have been developed by Thales SESO

- Simulations: optical models made to predict performances of objectives under cryogenic environment and also to as-built models at ambient temperature necessary for integration

- Manufacturing: each model is composed by 80 parts - mechanical and optical (including small lenses made with brittle crystalline structure materials like $\mathrm{ZnSe}$ ) - integrated with regards to tilt specifications (+/-50') and centering specifications $(+/-10 \mu \mathrm{m})$ wrt mechanical reference

- Coating: seventeen specific coatings in the infrared range have been designed and qualified under cryogenic and space environment for the different ranges and different substrates

- Testing: a new bench has been developed by Thales SESO in order to measure infrared WFE infrared performances of objectives under vacuum and cryogenic environments. Focal length and Line-of-Sight (LoS) are measured in ambient condition.

The engineering phase for the development of the four objectives lasted 4 years and consisted in the research of ultimate performances for each specifications, generating a high number of iterations, calculations, etc.

Conception is divided into three parallelized phases:

- The opto-mechanical with an objective to determine a solution for the lenses holding minimizing deformations of optical surfaces due to cryogenic environment. Those deformations are taken into account into the optical models in order to evaluate their impacts on performances (WFE, LoS...) 
- The optical tolerancing in order to take into account the lenses manufacturing and integration constraints. This phase induced lots of iterations with sub-contractors and integration teams. Tolerancing results participate to the assessment of the optical performances budgets.

- The optical surfaces coating design. The results of this design will be used for the assessment of spectral performances and straylight analysis.

\subsection{Optical conception}

Firstly, the choice of materials for lenses is important to address to the range of wavelength specifications. Materials used are the following and they have been characterized - in terms of optical properties and CTE - on the temperature and wavelength range:

- $\quad$ Fused Silica

- $\quad$ Silicon

- Germanium

- $\mathrm{ZnS}$

- $\mathrm{ZnSe}$

Then, the tolerancing specification, which is a critical point in the design, have been made in several steps and have a huge impact on the manufacturing. First step consist in taking into account all the manufacturing tolerances such as Radius of curvature, thickness, indexes, etc - in the ZEMAX model. Using Monte Carlo (MC) calculation, we have been able to generate a high number of possible manufacturing combination with performances associated for each of them. Considering the 3sigma statistical calculation from these MC, we got a robust model prediction of as-built final performances.

In a second step of optimization, this $\mathrm{MC}$ representative of manufacturing at 3 sigma is toleranced considering the integration parameters (lenses tilt and decentering). Finally, a new MC at 3 sigma is generated representative of both manufacturing and integration tolerancing.

The last optimization is performed after lenses manufacturing, where the measured parameters of each lens are injected in the ZEMAX model.

Based on that, it is possible to optimize the performances by modifying the following parameters, named compensators:

- Air gap between lenses

- Detectors lateral and axial position

\subsection{Opto-mechanical and mechanical conception}

Based on Thales SESO experience, the chosen solution for the lenses holding inside the barrel relies on the use of glue. Bonding lenses and barrel imply to choose wisely a glue operating under cryogenic environment. It is also needed to perfectly know or characterize the mechanical properties of the glue and the other optical and mechanical materials. Indeed, the opto-mechanical design phase is made using NASTRAN finite element calculations.

Thus, the first step is to characterize the silicon based glue chosen and the other materials (Titanium, Germanium, ZnSe, $\mathrm{ZnS}$, Silicon and fused silica) in terms of CTE. Measurement were made with a Michelson laser interferometer measurement system under vacuum. Traction tests have also been performed in order to determine the Young modulus and Yields stress of the glue after the vitreous transition temperature.

Then, the critical point of the opto-mechanical design was the glue thickness. Indeed, bonding will be made at ambient temperature, thus, the thickness need to be optimized in order to guarantee the mechanical resistance of the assembly while minimizing the stress induced in lenses and glue and minimizing the optical surface deformations when working at operating temperature. This determined thickness has to be a compromise between theoretical results obtained numerically and the manufacturing possibilities. 
Moreover, objectives work under vacuum, venting holes are needed in order to remove air and humidity present between the different lenses to prevent the apparition of steam or ice on the lenses during temperature variation.

Finally, based on those characterizations, finite element calculations are performed in order to determine and optimize the objectives design. Taking into account that the design is optimized for cryogenic environment, but, objectives are manufactured at ambient temperature. It is thus very important to anticipate the evolution of lenses curvature, lenses thickness and air gap on the design.

The opto-mechanical and optical conception are fully linked, the finite element modeling results being entries for ZEMAX calculations.

Additionally the mechanical surfaces of the objectives have a thermal contribution and relevant thermal coatings have been defined depending on the need : black coating for those surfaces needing high emissivity and protected gold coating on polished surfaces for the one needing very low emissivity.

Last some of the optics having a sealing function between the cryostat and the ambient atmosphere, Thales SESO also designed an air tight assembly for those components.

\subsection{Optical coating conception}

Due to the number of substrates materials and the number of spectral channels, seventeen antireflective (AR) coating designs operating in cryogenic conditions and under vacuum were developed.

This includes the 5 types of substrates (fused silica, silicon, germanium $\mathrm{ZnS}$ and $\mathrm{ZnSe}$ ) and the 4 spectral channels of MTG FCI (NIR, IR1, IR2 and IR3) and the two spectral ranges of MTG IRS (MWIR and LWIR).

Among the different designs some were covering a spectral bandwidth equivalent to their center wavelength, leading to designs with up to 16 layers for a single AR coating.

The main issue for these designs is related to ghost images. Any surface presenting a too high reflectance coefficient is potentially generating ghost images through multiple reflections inside the optical assembly.

Therefore, the reflectance is expected to be less than $1 \%$ over the full relevant wavelength range, with an average at about $0.5 \%$. These designs were calculated using Essential ${ }^{\circ}$ Software from Angus Mac Leod.

The optical coating designs went first through a trade-off to confirm that we were able to design and produce such sophisticated coatings.

Then, from this trade-off we ran a validation phase allowing to confirm the performances in corresponding ranges in the operating conditions (cryogenic temperature and vacuum). Impact of these operating conditions were evaluated and taken into account in the final designs of the different coatings. Impact of the substrates evolution when going to cryogenics was also taken into account after full characterization (basically the change in absorption of the substrate for some of them, when changing from room temperature to $80 \mathrm{~K}$ ). This demonstration was made using a dewar mounted on our spectrophotometers. Our material database for such ranges were updated from the measured results.

The other challenge was to prove the durability of these coatings. This was achieved first partly during the validation phase and then fully through the qualification phase.

The designed coatings were then demonstrated to survive without any degradation up to 150 cycles from ambient to $80 \mathrm{~K}$. This included verification on fully representative lenses, as mounted in their barrels and the objective main barrel.

\section{MANUFACTURING}

Each model is composed by 80 parts - mechanical and optical (small lenses made with brittle materials such as $\mathrm{ZnSe}$ ) integrated complying with tilt specifications $(+/-50$ ') and centering specifications $(+/-10 \mu \mathrm{m})$. Moreover, in order to ensure the mechanical resistance generated by dynamic loads and generate symmetrical optical surface deformation, the glue joint need to be continuous. This need implies constraints in terms of logistics, integration, lenses manipulation, optical measurement. 
Materials used are very fragile, particularly ZnSe. Micro-cracks need to be avoided to limit crack propagation during temperature changes. Thus, very detailed inspection, manipulation and storage of lenses have been additional constraints.

To reach the tilt, centering specifications two optical centering tools have been developed in order to first bond the lens inside its barrel, then the couple lens/barrel inside the main barrel of the objective.

Those bonding in the main barrel are made in collaboration with engineering team. Indeed, based on the asmanufactured lenses, air gaps at ambient temperature are precisely calculated from optimization under cryogenic environment.
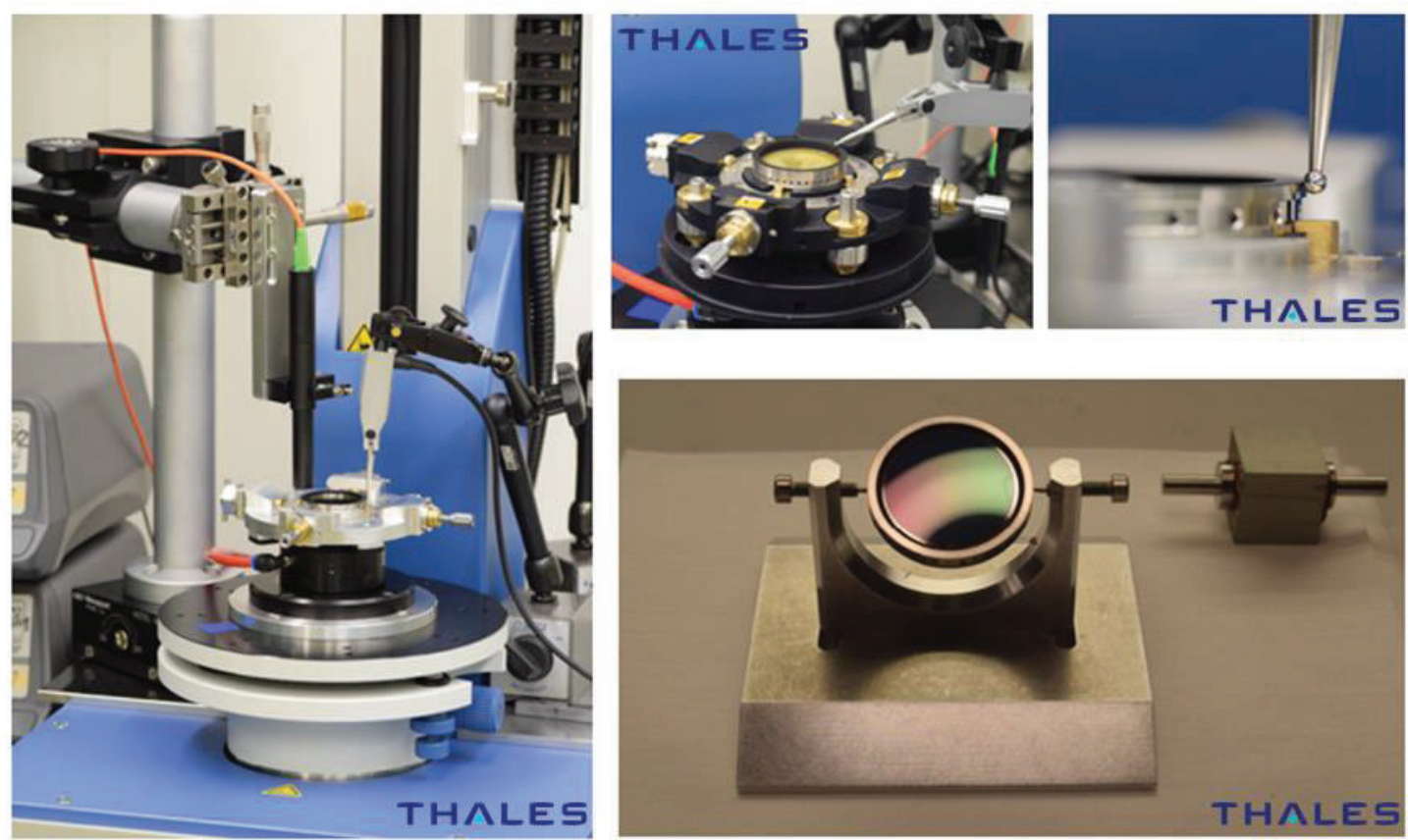

Figure 3-1: view of the integration bench

\section{TEST, MEASUREMENT AND RESULTS}

After integration, objectives have been mechanically qualified by being submitted to dynamic and thermal environments:

- $\quad 12$ gRMS in random

- $\quad 50 \mathrm{~g}$ in sine

- $\quad$ Thermal cycle between $75 \mathrm{~K}$ and $313 \mathrm{~K}$

Measurement at ambient temperature and under cryogenic environment are performed at THALES SESO facilities. A bench has been especially developed for those measurement using PHASICS sensor.

The PHASICS bench has been specially developed to answer the WFE measurements needs of MTG-Cold Optics program. The bench is composed of two measurements wavelength $(3.39 \mu \mathrm{m}$ and $10.6 \mu \mathrm{m})$ and one alignment wavelength $(632.8 \mathrm{~nm})$. Each IR wavelength can provide two exit pupil thanks to specific collimator. 


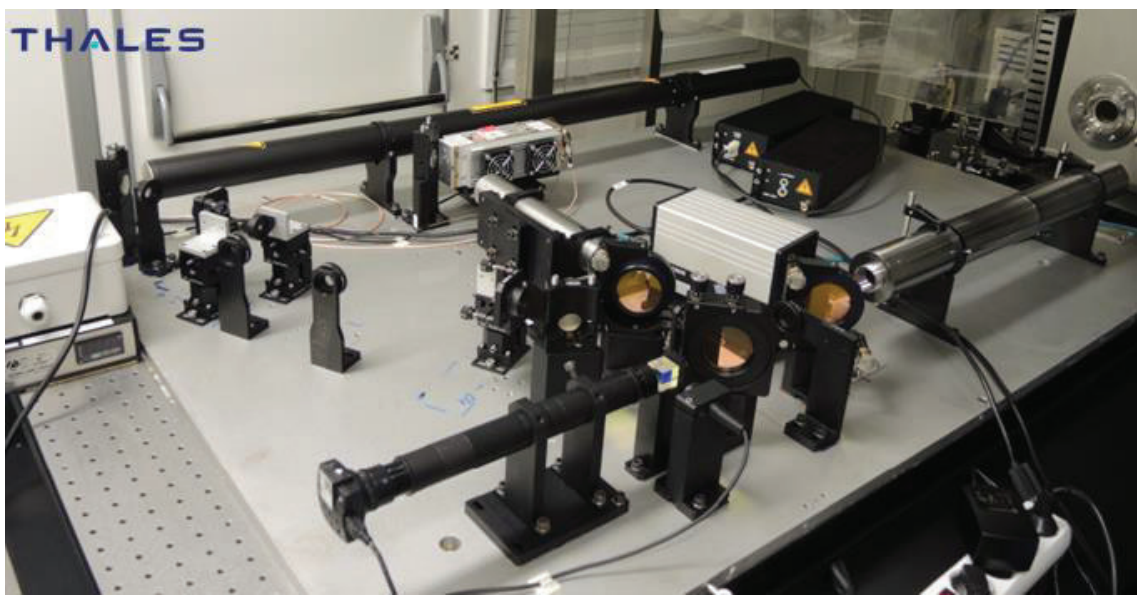

Figure 4-1: View of the PHASICS bench

The transmitted WFE under vacuum and cryogenic environment is between $100 \mathrm{~nm}$ RMS and 250nm RMS in the range of $1 \mu \mathrm{m}$ to $14 \mu \mathrm{m}$. Measurement are in accordance with the ZEMAX optical model used to define the staking of lenses.

Line-of-sight is better than $0.1^{\circ}$ in both axis, confirming the good integration of lenses into the main barrel.

Focal length measurements are coherent with theoretical performance from ZEMAX optical model, better than measurement accuracy of $0.4 \%$.

Spectral transmission is compliant to requirement, thanks to coatings with reflectance around $0.5 \%$ on the spectral band.

\section{CONCLUSION}

Design cryogenic objectives is a complex iterative work requiring lots of interaction between mechanical engineer, optical engineer, coating engineer and integration engineer. Integration of the objectives required to develop specific and dedicated bench in order to reach the specifications. Moreover, a cryogenic vacuum chamber has been developed in order to perform the measurement of optical performances under cryogenic environment. The major results show the adequacy between measurement and theoretical performances assessed using predictive optical models. These results have validated our method of conception and integration.

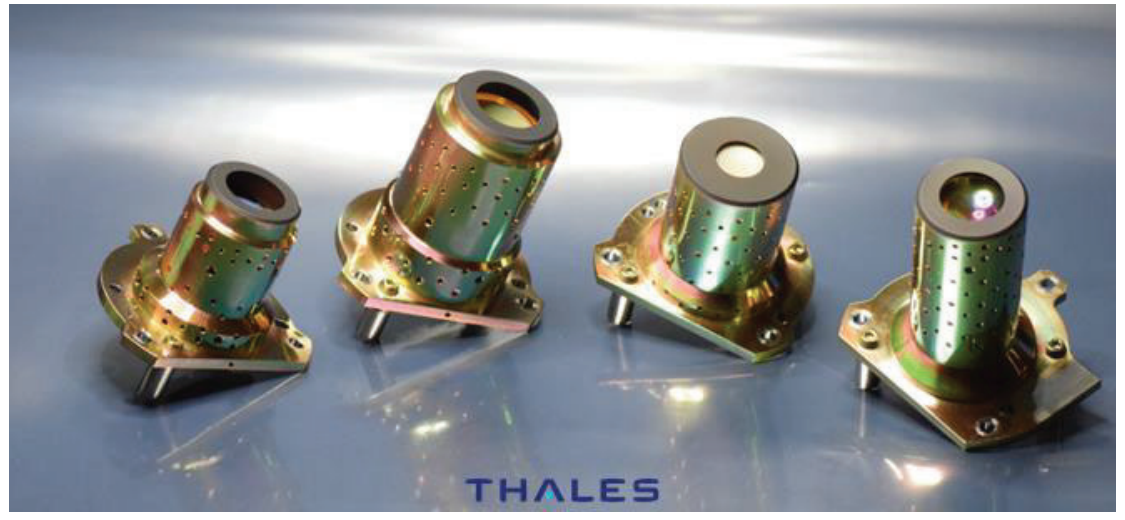

Figure 5-1: Picture of the four MTG Cold Optics Imager objectives

\section{ACKNOWLEDGEMENTS}

The work for the Meteosat Third Generation (MTG) Programme has been performed under contract from the European Space Agency (ESA) with OHB and Thales Alenia Space. 